\title{
Growing Critical: Self-Organized Criticality in a Developing Neural System
}

\author{
Felipe Yaroslav Kalle Kossio, ${ }^{1}$ Sven Goedeke, ${ }^{1}$ Benjamin van den Akker, ${ }^{2}$ \\ Borja Ibarz, ${ }^{3}$ and Raoul-Martin Memmesheimer ${ }^{1,2}$ \\ ${ }^{1}$ Neural Network Dynamics and Computation, Institute of Genetics, University of Bonn, Bonn, Germany \\ ${ }^{2}$ Department of Neuroinformatics, Radboud University Nijmegen, Nijmegen, Netherlands \\ ${ }^{3}$ Nonlinear Dynamics and Chaos Group, Departamento de Fisica, Universidad Rey Juan Carlos, Madrid, Spain
}

(Received 17 November 2017; revised manuscript received 15 May 2018; published 3 August 2018)

Experiments in various neural systems found avalanches: bursts of activity with characteristics typical for critical dynamics. A possible explanation for their occurrence is an underlying network that selforganizes into a critical state. We propose a simple spiking model for developing neural networks, showing how these may "grow into" criticality. Avalanches generated by our model correspond to clusters of widely applied Hawkes processes. We analytically derive the cluster size and duration distributions and find that they agree with those of experimentally observed neuronal avalanches.

DOI: 10.1103/PhysRevLett.121.058301

Introduction.-A hallmark of systems at criticality is the variability of their responses to small perturbations. While small responses are most likely, the probability of large, system-size effects is non-negligible. Various natural and model complex systems show similar behavior [1]. One explanation is that they drive themselves close to a critical state ("self-organized criticality" [2,3]). The dynamics of such systems are characterized by "events" or "avalanches." Their sizes and durations follow power-law distributions, frequently with exponents $3 / 2$ and 2 , indicating an underlying critical branching process [4-7]. Apparent critical dynamics, "neuronal avalanches," in biological neural networks were first reported in Refs. [8,9]. It has been suggested that they foster information storage and transfer $[10,11]$. Experimental studies often report power-law size and duration distributions with exponents $3 / 2$ and 2 . They further indicate that neuronal avalanches emerge during development [12-15], suggesting that neural networks develop into a critical state.

The development of neural networks is determined by an interplay of genetic determinants and environmental influence. Of pivotal importance is neural activity [16,17]. As a general rule, neurons with low activity level extend their neurites and form more activating connections, while highly active cells reduce these [18-20]. Thereby, neurons maintain their average activity at a particular level (homeostasis) [21-23].

Computational models for avalanches in neural systems rely on static, tuned connectivity [14,24], on short-term synaptic plasticity $[25,26]$, or on long-term network changes [27-30]. Here we propose a continuous-time spiking neural network model belonging to the third class. The avalanche dynamics follow from a network growth process towards a critical state, which uses local information only [31-33]. The model is rooted in previous models for neural network development [27,29,34], but sufficiently simple to be analytically tractable.

Neuron model.-Like biological neurons, our model neurons communicate by sending and receiving spikes in continuous time. Spiking is stochastic, according to an inhomogeneous Poisson point process with instantaneous rate $f_{i}(t)$ for neuron $i$ [27,35-38]. In isolation neurons have a low spontaneous rate $f_{0}$, e.g., due to spontaneous synaptic release or channel fluctuations $[39,40]$. A spike from neuron $j$ increases $f_{i}$ by the size of the time-dependent connection strength $g A_{i j}$. The increment decays exponentially with time constant $\tau$, which accounts for relaxation due to leak currents. The couplings are excitatory; this is the dominant connection type in developing neural systems [34]. Taken together, $f_{i}$ 's dynamics follow

$$
\tau \dot{f}_{i}(t)=f_{0}-f_{i}(t)+\tau g \sum_{j} A_{i j}\left(t^{-}\right) \sum_{\hat{t}_{j}} \delta\left(t-\hat{t}_{j}\right),
$$

where $\hat{t}_{j}$ denotes the spike times of neuron $j$ ( $\delta$ is the Dirac delta distribution). For simplicity, we assume that all neurons have the same parameters. For constant couplings, the network dynamics form a multivariate Hawkes point process $[37,38,41]$.

Network growth.-Neurons are commonly arranged in single or stacked layers. We thus represent neurite extents by disks with radii $R_{i}(t)$, with centers, representing cell somas, randomly and uniformly distributed in a planar area $[27,34,42]$. Since neurons with more neurite overlap can grow more synaptic connections [43,44], connection strengths are set proportional to the overlap areas $A_{i j}(t)$ of the disks, with proportionality constant $g$. We incorporate homeostatic neurite growth by evolving extents as 


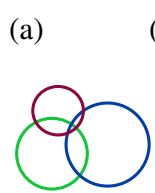

(b)

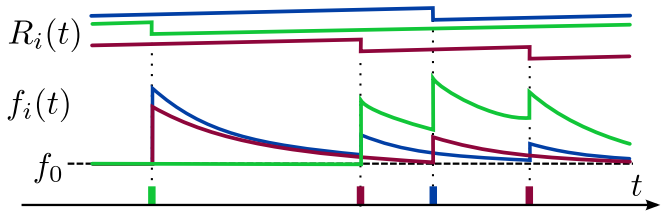

FIG. 1. Neuron dynamics. (a) Neurons' somas and neurite extents are represented by disks with evolving radii. Coupling strengths are proportional to neurite overlap areas. (b) Neurite radii increase linearly (upper traces), own spike sendings (lower trace) result in instantaneous shrinkage. Spike arrivals increase the instantaneous firing rate by the coupling strength, it decays exponentially in between (middle trace).

$$
\dot{R}_{i}(t)=K\left(1-\frac{1}{f_{\text {sat }}} \sum_{\hat{t}_{i}} \delta\left(t-\hat{t}_{i}\right)\right),
$$

Fig. 1. Between spikes of neuron $i, R_{i}(t)$ grows linearly with rate $K$. At spike sending, it shrinks by a constant amount $K / f_{\text {sat }}$, which determines the rate $f_{\text {sat }}$ at which growth and shrinkage equilibrate. There are no selfconnections. Growth takes much longer than decay of activity, $1 / K \gg \tau$ (spatial scales of the population are of order one). Furthermore, we assume $f_{\text {sat }} \gg f_{0}$, in agreement with experiments $[15,40,45]$. Spontaneously inactive neurons would reduce the relevant average $f_{0}$ [45]. The growth model is biologically inspired; it is a simplification of previous growth models [23,27,29,34]. However, many slow homeostatic processes $[21,22,48]$ with $f_{\text {sat }} \gg f_{0}$ will yield similar results.

The neurons are initially mostly isolated. Over time, they extend their neurites, form connections, and develop a network, Fig. 2. At intermediate stages, neurites and overlaps can overshoot $[15,29,34]$. When neuron $i$ 's time-averaged firing rate $\bar{f}_{i}$ reaches $f_{\text {sat }}$, its average growth stops [Eq. (2)]. Our networks grow into a stationary state, where $\bar{f}_{i}=f_{\text {sat }}$ for all $i$. In the following, we investigate this state.

Stationary state dynamics.-We first compute the average number of spikes that a spike directly causes: Identical $\bar{f}_{i}$ imply identical time-averaged total overlaps $\sum_{j} \bar{A}_{i j}=$ $\bar{A}_{i}=\bar{A}$ and input coupling strengths. Time averaging Eq. (1), $\bar{f}_{i}=f_{0}+\tau g \sum_{j} \bar{A}_{i j} \bar{f}_{j}$ [here and henceforth we neglect the small fluctuations of $A_{i j}(t)$ around $\left.\bar{A}_{i j}\right]$, and inserting $f_{\text {sat }}$ yields $\tau g \bar{A}=1-f_{0} / f_{\text {sat }}$. A spike of neuron $j$ at $\hat{t}_{j}$ adds $g A_{i j}\left(\hat{t}_{j}^{-}\right) e^{-\left(t-\hat{t}_{j}\right) / \tau} \Theta\left(t-\hat{t}_{j}\right)$ to $f_{i}(t)$ [Eq. (1), $\Theta$ is the Heaviside function], such that the number of additionally induced spikes in neuron $i$ is Poisson distributed with mean $\tau g A_{i j}\left(\hat{t}_{j}^{-}\right)$. Averaged over the randomness of spike generation each spike thus generates in total

$$
\sigma=\tau g \sum_{i} \bar{A}_{i j}=\tau g \bar{A}=1-\frac{f_{0}}{f_{\text {sat }}}
$$

(a)
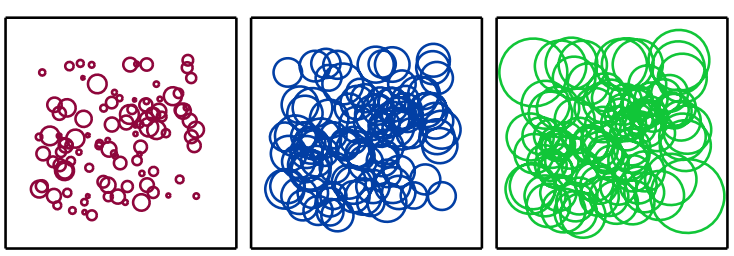

(b)
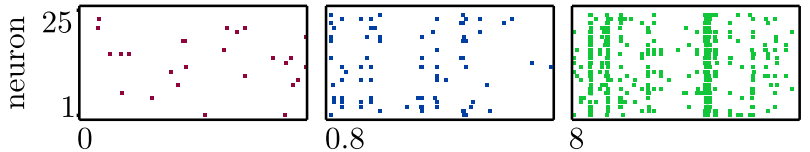

(c)

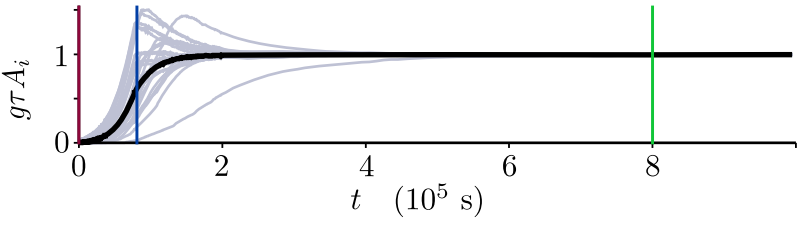

FIG. 2. Network dynamics. (a) Extents of neurites. (b) Spikes generated by 25 sample neurons (100 s windows). (c) Scaled total overlaps of 25 sample neurons (gray) and the population average (black) as a function of time. For (a) and (b) from left to right: initial state (red), state with growth on average (blue), stationary state (green). Color coded vertical lines in (c) indicate the three different time points in (a) and (b).

spikes, where we used the symmetry of overlaps, $A_{i j}=A_{j i}$, $\sum_{i} \bar{A}_{i j}=\sum_{j} \bar{A}_{i j}=\bar{A}$. Equation (3) holds independently of network activity and neuron identity, due to the linearity of Eq. (1) and the homogeneity of parameters. In particular, $\sigma$ equals also the time and population average number of induced spikes $\left(\propto f_{\text {sat }}-f_{0}\right)$ per spike $\left(\propto f_{\text {sat }}\right)$.

The independence of spike offspring generation from other spikes allows us to understand the dynamics as a branching process with branching parameter $\sigma$. More specifically, we have an age-dependent or Crump-Mode-Jagers branching process [49]: Individuals (spikes) generate offspring at an age-dependent rate. Neuronal avalanches are trees of offspring, started by a spontaneous spike. For their overall size only the distribution of single spike offspring matters. It is Poissonian with parameter $\sigma$. The avalanche sizes $s$ therefore follow the Borel distribution [50],

$$
P(s)=\frac{(s \sigma)^{s-1} e^{-s \sigma}}{s !} .
$$

We apply Stirling's approximation to obtain

$$
P_{\mathrm{appr}}(s)=\frac{1}{\sqrt{2 \pi} \sigma} s^{-3 / 2} e^{-(\sigma-\ln \sigma-1) s},
$$

explicitly highlighting the power-law tail with exponent $3 / 2$ of a critical branching process for $\sigma=1$ [4-7]. For a subcritical process $(\sigma<1)$, Eq. (5) is a power law with exponential cutoff around $s_{c}(\sigma)=(\sigma-\ln \sigma-1)^{-1}$. It signifies subcritical dynamics [4,5,51], not a finite size effect $[3,5]$; the size distribution is independent of neuron number. 
Equation (5) inherits the good quality of Stirling's approximation [52], with relative error about $1 /(12 s)$.

The heights of Crump-Mode-Jagers trees, i.e., the temporal differences $T$ between their first and last individuals, represent the durations of the corresponding neuronal avalanches. In the following we derive their probability density $p(T)$. Because of the additivity of Poisson processes, the superposition of all neurons' spike trains can be described as an inhomogeneous Poisson process with rate $f(t)=\sum_{i} f_{i}(t)$. Summing Eq. (1) over $i$ and inserting $\bar{A}=\sigma /(g \tau)$ [Eq. (3)] yields $\tau \dot{f}(t)=N f_{0}-$ $f(t)+\sigma \sum_{\hat{t}} \delta(t-\hat{t})$ with the number of neurons $N . \hat{t}$ are the neurons' spike times; they occur with instantaneous rate $f(t)$. The spiking dynamics may thus be interpreted as a self-exciting Hawkes process. It is Markovian due to the exponentially decaying impact kernel $[53,54]$. The spontaneous background rate $N f_{0}$ initiates avalanches. To determine their durations, we consider an analogous process with instantaneous rate $f_{a}(t)$ and without spontaneous spiking, which is initiated at $t=0$ by an external spike,

$$
\tau \dot{f}_{a}(t)=-f_{a}(t)+\sigma \sum_{\hat{t}_{a}} \delta\left(t-\hat{t}_{a}\right), f_{a}(0)=\frac{\sigma}{\tau} .
$$

The duration of an avalanche is the time $T$ of this process' last spike. The probability that it has occurred before $t$ gives the distribution function $P(T \leq t)$ of durations. We first compute this probability conditioned on the instantaneous rate $f_{a}(t)$ at the end of the considered interval:

$$
\begin{aligned}
P\left(T \leq t \mid f_{a}(t)\right) & =P\left(\text { no spike in }(t, \infty) \mid f_{a}(t)\right) \\
& =e^{-\int_{t}^{\infty} f_{a}\left(t^{\prime}\right) d t^{\prime}}=e^{-\tau f_{a}(t)},
\end{aligned}
$$

where we use that the process behaves like a Poisson process with exponentially decaying rate, if no spike is generated. Averaging over $f_{a}(t)$ yields

$$
\begin{aligned}
P(T \leq t) & =\int_{0}^{\infty} P\left(T \leq t \mid f_{a}(t)\right) p\left(f_{a}(t)\right) d f_{a}(t) \\
& =E\left(e^{-\tau f_{a}(t)}\right) .
\end{aligned}
$$

$E(\cdot)$ denotes the expectation value over the process, Eq. (6). Importantly, Eq. (8) shows that $P(T \leq t)$ equals the Laplace transform of the random variable $f_{a}(t)$, evaluated at the decay time $\tau$. This Laplace transform has recently been derived [55-57]. Inserting our parameters yields $E\left(e^{-\tau f_{a}(t)}\right)=e^{\sigma a(t) / \tau}$, where $a(t)$ satisfies

$$
\dot{a}(t)=-a(t) / \tau+e^{\sigma a(t) / \tau}-1, \quad a(0)=-\tau .
$$

The resulting $P(T \leq t)=e^{\sigma a(t) / \tau} \Theta(t)$ with $\Theta(0)=1$ has the density

$$
p(T)=\sigma \dot{a}(T) e^{\sigma a(T) / \tau} \Theta(T) / \tau+e^{-\sigma} \delta(T) .
$$

We can generalize Eq. (9) to Hawkes processes with different kernels using the integral equation for cluster duration distributions [58,59].

We finally approximate $p(T)$ by closed-form expressions with a focus on its tail near criticality. For large $t$, $P(T \leq t)$ approaches 1, so $a(t)$ approaches 0. Generally, $\sigma a(t) / \tau$ stays between -1 and 0 . Expanding $e^{\sigma a(t) / \tau}$ in Eq. (9) around $\sigma a(t) / \tau=0$ to second order,

$\dot{a}(t) \approx(\sigma-1) a(t) / \tau+\sigma^{2} a(t)^{2} /\left(2 \tau^{2}\right), \quad a(0)=-\tau$,

yields closed-form approximations for $a(t)$. In particular, for nearly critical systems with $\sigma \approx 1$, the first term on the right-hand side vanishes and the solution is $a_{\text {appr }}(t)=$ $-(2 \tau)^{2} /(2 \tau+t)$, leading to a probability density

$p_{\text {appr }}(T)=2 \tau(2 \tau+T)^{-2} e^{a_{\text {apr }}(T) / \tau} \Theta(T)+e^{-1} \delta(T)$,

which approaches for large $T$ a power law with critical exponent 2. For large $t$ the error in the expansion Eq. (11) becomes negligible, $a_{\text {appr }}(t)$ thus has the right slope and $p_{\text {appr }}(T)$ equals $p(T)$ up to a factor (Fig. 3). We conclude that the duration distribution has a power-law tail with critical exponent 2. Expanding the exponential to third order yields a closed-form distribution that is a good approximation also for small $T$.

Simulations.-We complement our analytics with simulations to (i) compare the avalanche distributions, (ii) exemplify the irrelevance of connectivity fluctuations, (iii) investigate the spatial spread of avalanches, (iv) address the robustness of the results, and (v) consider a typical experimental manipulation. If not stated otherwise,
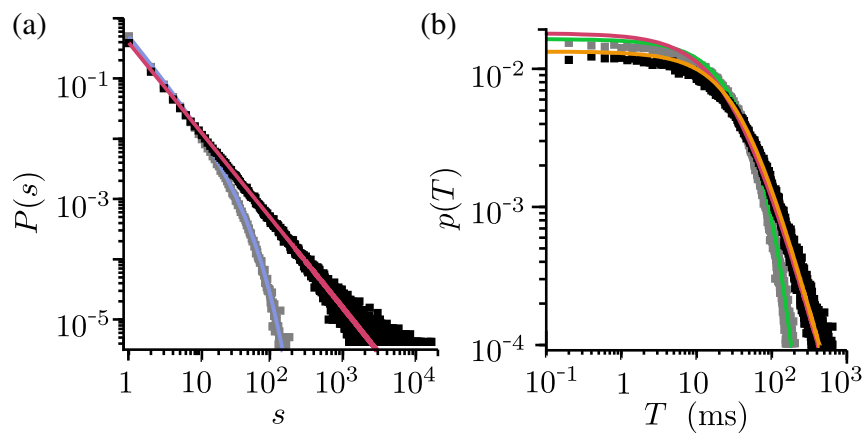

FIG. 3. Avalanche sizes and durations. (a) Analytical size distributions Eq. (5) (discrete points connected) and simulation results for subcritical $\left(f_{\text {sat }}=0.04 \mathrm{~Hz}, \sigma=0.75, t_{\text {bin }}=30 \mathrm{~ms}\right.$, blue and gray) and near-critical $\left(f_{\text {sat }}=2 \mathrm{~Hz}, \sigma=0.995, t_{\text {bin }}=\right.$ $45 \mathrm{~ms}$, red and black) states. Equation (4) yields visually indistinguishable analytics. (b) Analytical duration distributions Eq. (10) and simulation results, for subcritical (green and gray) and near-critical (orange and black) states, and closed-form approximation Eq. (12) (red). 
$N=100, \tau=10 \mathrm{~ms}[60], g=500 \mathrm{~Hz}, f_{0}=0.01 \mathrm{~Hz}$, $f_{\text {sat }}=2 \mathrm{~Hz}[15,40]$, somas are placed on unit square, $K^{-1}=10^{6} \mathrm{~s}$ (quick growth, accelerating simulations) $[15,21,22,29]$. The simulations use an event-based algorithm. Next spike times are determined using inverse transform sampling of the interspike-interval distribution; we avoid nonelementary functions by splitting each neuron's Poisson process into a homogeneous (rate $f_{0}$ ) and an inhomogeneous one.

An avalanche should be a sequence of offspring spikes of one spontaneous progenitor. To keep contact with the experimental literature, we analyze numerical data by binning time and considering spike sequences that are not separated by an empty bin as one avalanche [8,61-63]. Our model yields analytical estimates for the probabilities that binning splits the first avalanche spikes or merges them with the next avalanche, as well as for splitting or merging an average avalanche. Keeping them moderate provides our bin sizes $t_{\text {bin }}$ in terms of experimentally accessible quantities $\left(f_{0}, \tau, N, f_{\text {sat }}\right)$ [45]. Results are robust against changing $t_{\text {bin }}$.

(i) In all simulations the model reaches a stationary state. The avalanche distributions agree well with the analytically derived ones, Fig. 3, the effects of binning and avalanche overlaps are small. We quantitatively test this agreement using the methods described in Refs. [64,65]. For both size and duration distributions a pure power law is ruled out, as expected. For the size distribution, a power law with exponential cutoff, cf. Eq. (5), yields a good fit. The analytical values of the power-law exponent, the cutoff $s_{c}(\sigma)$, and the resulting $\sigma$ are closely matched.

(ii) The fluctuations of $\sum_{j} A_{i j}(t)$ and the deviations of $\bar{f}_{i}$ from $f_{\text {sat }}$ are small $(<1 \%$, Fig. 2). Freezing the network $(K=0)$ in the stationary state has no effect on the avalanche statistics: Neuronal growth carries the system close to a critical point, but is not required later on. This is in agreement with self-organized criticality and excludes other mechanisms [66-68].

(iii) To investigate spatial spread near criticality, we compute covariances $C_{i j}=\left\langle n_{i} n_{j}\right\rangle-\left\langle n_{i}\right\rangle\left\langle n_{j}\right\rangle$ between numbers $n_{i}, n_{j}$ of spikes contributed to single avalanches by neurons $i, j$ with various distances. Covariances decay comparably slowly. Covariances and thus avalanches spread beyond direct connections, Fig. 4(a).

(iv) To test robustness, we first freeze the growth in the stationary state and shuffle the output vectors (columns of the coupling matrix) between neurons. While this alters the network topology and breaks coupling symmetry, it leaves the essential total coupling strengths unchanged. Indeed, we observe little effect on avalanche sizes and durations. Second, we consider moderate nonadditivity of spike impacts. We introduce an absolute refractory period $\tau_{\text {ref }}$ after a sent spike, during which the neuron cannot spike again. We observe that although the refractory period limits the firing rate, the network reaches a stationary state with
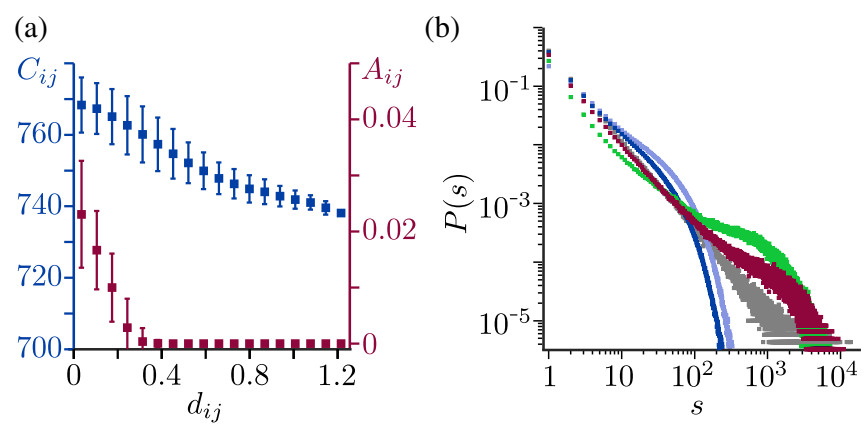

FIG. 4. (a) Pairwise spike number covariances and overlaps as functions of the intersomatic distances $d_{i j}$ (averages around a particular intersomatic distance, bars: standard deviations), $\sigma=0.995, t_{\text {bin }}=45 \mathrm{~ms}$. (b) Avalanche size distributions for the same model as in Fig. 3, but with absolute refractory period $\tau_{\text {ref }}=\tau$ (red), $\tau_{\text {ref }}=4 \tau$ (green), $\tau_{\text {ref }}=0 \mathrm{~ms}$ (gray) for reference, $t_{\text {bin }}=45 \mathrm{~ms} ; \tau=5 \mathrm{~ms}, \tau_{\text {ref }}=4 \tau, f_{0}=0.1 \mathrm{~Hz}, f_{\text {sat }}=0.8 \mathrm{~Hz}$, $t_{\text {bin }}=10 \mathrm{~ms}$ (blue, $t_{\text {bin }}=45 \mathrm{~ms}$ : light blue).

the same average individual rate $f_{\text {sat }}$ as before: larger overlaps compensate refractoriness. For a refractory period about $\tau$, which is often biologically plausible $[39,60]$, the statistics resemble the original one for small and medium size avalanches [Fig. 4(b), red vs. gray]. Larger couplings and stop of avalanches lacking available neurons cause an excess of larger avalanches, followed by a strong reduction. Neurons still frequently contribute several spikes to avalanches. With long refractoriness, little similarity remains [Fig. 4(b), green and blue; blue: our model with parameters adapted from Ref. [27], calcium variable present in Ref. [27] does not affect distribution shape].

(v) Manipulation of neural excitability or coupling strength via $g$ causes subcriticality (decreased $g$ ) or excess of large avalanches (increased $g$ ), as in experiments $[8,69]$. Our model predicts that the latter is balanced by network plasticity faster than the former, due to strongly increased activity [45].

Discussion and conclusion.-We suggest an analytically tractable model for neural network growth, which may explain the emergence of subcritical and critical avalanche dynamics. It covers essential features of biological neurons such as operation in continuous time, spiking, leak currents, and network growth. Still, it allows the analytical computation of the avalanche size and duration distributions for subcritical and critical stationary states. Our numerical analysis confirms their validity and robustness and yields additional insight.

Two features are responsible for the emergence of the (near-)critical state (Fig. 3): (i) homeostatic growth to attain a saturation rate that is high compared to the spontaneous one (precise values of $f_{\text {sat }}$ and $f_{0}$ are irrelevant), and (ii) linear summation of spike impacts. (i) implies that in the stationary state on average each spike generates nearly one successor. This holds for all networks with largely selfsustained activity. Usually, however, branching parameters 
vary, for example at high network activity spikes generate less offspring. This drives activity excursions back and generates non-power-law distributions [70,71]. In our networks, (ii) implies that the branching parameter is the same for each spike. Small saturation rates yield subcritical dynamics (Fig. 3), strong nonlinearities other deviations [Fig. 4(b)]. Our model thus predicts that neural networks may develop criticality already due to their growth, that spontaneous spiking in such networks is low compared to saturation, and that spike effects add rather linearly and are independent of activity level. For example, starburst amacrine cells have radial dendritic trees, interact during development via dendro-dendritic excitatory connections, and are reported to generate critical avalanches [14]. Our model predicts that higher precision measurements will reveal deviations as in Fig. 4(b), due to the cells' long refractory periods.

Our network model is based on the neurobiologically more detailed ones $[27,29,34]$. Motivated by experiments, Ref. [34] proposes radial activity dependent neurite outgrowth steered by calcium dynamics and finds convergence to a stationary state for certain parameter ranges. To study avalanches, Ref. [27] adds stochastically spiking neurons, albeit with long refractoriness and larger $f_{0} / f_{\text {sat }}$, impeding analytical treatment and causing large deviations from criticality [Fig. 4(b)]; Ref. [29] assumes antagonistic growth of axons and dendrites and finds criticality, if a certain fraction of neurons becomes inhibitory; Refs. [48,72,73] consider more abstract homeostasis and neuron models.

Usually, models for neuronal avalanches only allow to estimate size and duration distributions numerically [14,25,27,29,30]. Reference [24] obtains an analytical expression of the size distribution for a discrete-time network. Our article derives size and duration distributions for a continuous-time spiking network model after selforganization. These distributions depend only on the experimentally accessible parameters $f_{0} / f_{\text {sat }}$ and $\tau$ (duration scaling). The power-law exponents agree with experimentally found ones and those of simple branching processes $[4,6,8,14]$. The duration distribution has power-law scaling at the tail $[4,6,14]$, a fit to short avalanches [8] would yield different results. Our analytical expressions allow fast parameter scans, delineations of the (near-)critical regime and parameter estimations.

From a general perspective, avalanches in our model are clusters of a Hawkes process. While their size distribution can be straightforwardly computed [Eq. (4)], their duration distribution generally requires solving a nonlinear integral equation [58,59]. Here we show that for Markovian Hawkes processes it follows from the solution of an ordinary differential equation [Eqs. (9), (10)] and we give closed-form approximations. This may find straightforward application in further fields of science where these processes are employed, for example, to characterize durations of financial market fluctuations [57], earthquakes [74], violence [75], and epidemics [76,77].
We thank Matthias Hennig, Anna Levina, Viola Priesemann, and Johannes Zierenberg for helpful discussions and the German Federal Ministry of Education and Research (BMBF) for support via the Bernstein Network (Bernstein Award 2014, 01GQ1501 and 01GQ1710).

[1] D. Marković and C. Gros, Phys. Rep. 536, 41 (2014).

[2] P. Bak, C. Tang, and K. Wiesenfeld, Phys. Rev. Lett. 59, 381 (1987).

[3] P. Bak, C. Tang, and K. Wiesenfeld, Phys. Rev. A 38, 364 (1988).

[4] S. Zapperi, K. B. Lauritsen, and H. E. Stanley, Phys. Rev. Lett. 75, 4071 (1995).

[5] H. Jensen, Self-Organized Criticality (Cambridge University Press, Cambridge, 1998).

[6] T. E. Harris, The Theory of Branching Processes (Courier, New York, 2002).

[7] S. di Santo, P. Villegas, R. Burioni, and M. A. Muñoz, Phys. Rev. E 95, 032115 (2017).

[8] J. Beggs and D. Plenz, J. Neurosci. 23, 11167 (2003).

[9] J. Beggs and D. Plenz, J. Neurosci. 24, 5216 (2004).

[10] C. Haldeman and J. M. Beggs, Phys. Rev. Lett. 94, 058101 (2005).

[11] W. L. Shew and D. Plenz, Neurosci. 19, 88 (2013).

[12] A. Mazzoni, F. Broccard, E. Garcia-Perez, P. Bonifazi, M. Ruaro, and V. Torre, PLoS One 2, e439 (2007).

[13] E. Gireesh and D. Plenz, Proc. Natl. Acad. Sci. U.S.A. 105, 7576 (2008).

[14] M. H. Hennig, C. Adams, D. Willshaw, and E. Sernagor, J. Neurosci. 29, 1077 (2009).

[15] Y. Yada, T. Mita, A. Sanada, R. Yano, R. Kanzaki, D. J. Bakkum, A. Hierlemann, and H. Takahashi, Neuroscience (N.Y.) 343, 55 (2017).

[16] S. Kater and L. Mills, J. Neurosci. 11, 891 (1991).

[17] S. Kater, M. Mattson, C. Cohan, and J. Connor, Trends Neurosci. 11, 315 (1988).

[18] C. Cohan and S. Kater, Science 232, 1638 (1986).

[19] F. van Huizen and H. Romijn, Brain Res. 408, 271 (1987).

[20] R. Fields, E. Neale, and P. Nelson, J. Neurosci. 10, 2950 (1990).

[21] A. van Ooyen, Nat. Rev. Neurosci. 12, 311 (2011).

[22] G. Turrigiano, Cold Spring Harbor Perspect. Biol. 4, a005736 (2012).

[23] A. van Ooyen and M. Butz-Ostendorf, The Rewiring Brain (Academic Press, London, 2017).

[24] C. W. Eurich, J. M. Herrmann, and U. A. Ernst, Phys. Rev. E 66, 066137 (2002).

[25] A. Levina, J. M. Herrmann, and T. Geisel, Nat. Phys. 3, 857 (2007)

[26] A. Levina, J. M. Herrmann, and T. Geisel, Phys. Rev. Lett. 102, 118110 (2009).

[27] L. Abbott and R. Rohrkemper, Prog. Brain Res. 165, 13 (2007).

[28] V. Gómez, A. Kaltenbrunner, V. López, and H. J. Kappen, Adv. Neural Inf. Process. Syst. 21, 513 (2009).

[29] C. Tetzlaff, S. Okujeni, U. Egert, F. Wörgötter, and M. Butz, PLoS Comput. Biol. 6, e1001013 (2010). 
[30] B. Del Papa, V. Priesemann, and J. Triesch, PLoS One 12, e0178683 (2017).

[31] S. Bornholdt and T. Rohlf, Phys. Rev. Lett. 84, 6114 (2000).

[32] S. Bornholdt and T. Röhl, Phys. Rev. E 67, 066118 (2003).

[33] C. Meisel and T. Gross, Phys. Rev. E 80, 061917 (2009).

[34] A. van Ooyen and J. van Pelt, J. Theor. Biol. 167, 27 (1994).

[35] W. Gerstner and W. Kistler, Spiking Neuron Models: Single Neurons, Populations, Plasticity (Cambridge University Press, Cambridge, 2002).

[36] R. Kempter, W. Gerstner, and J. L. van Hemmen, Phys. Rev. E 59, 4498 (1999).

[37] A. Burkitt, M. Gilson, and J. L. van Hemmen, Biol. Cybern. 96, 533 (2007).

[38] V. Pernice, B. Staude, S. Cardanobile, and S. Rotter, PLoS Comput. Biol. 7, e1002059 (2011).

[39] C. Koch, Biophysics of Computation (Oxford University Press, Oxford, 1999).

[40] K. J. Ford, A. L. Félix, and M. B. Feller, J. Neurosci. 32, 850 (2012).

[41] A. G. Hawkes, Biometrika 58, 83 (1971).

[42] J. Barral and A. Reyes, Nat. Neurosci. 19, 1690 (2016).

[43] M. Abeles, Corticonics: Neural Circuits of the Cerebral Cortex (Cambridge University Press, Cambridge, 1991).

[44] A. van Ooyen, A. Carnell, S. de Ridder, B. Tarigan, H. D. Mansvelder, F. Bijma, M. de Gunst, and J. van Pelt, PLoS One 9, e85858 (2014).

[45] See Supplemental Material at http://link.aps.org/ supplemental/10.1103/PhysRevLett.121.058301 for further details, which includes Refs. [46,47].

[46] S. Johansson and P. Arhem, Proc. Natl. Acad. Sci. U.S.A. 91, 1761 (1994).

[47] J. Zheng, S. Lee, and Z. Zhou, Nat. Neurosci. 9, 363 (2006).

[48] J. Zierenberg, J. Wilting, and V. Priesemann, Phys. Rev. X 8, 031018 (2018).

[49] K. S. Crump and C. J. Mode, J. Math. Anal. Appl. 24, 494 (1968).

[50] J. C. Tanner, Biometrika 48, 222 (1961).

[51] V. Priesemann, M. Wibral, M. Valderrama, R. Pröpper, M. Le Van Quyen, T. Geisel, J. Triesch, D. Nikolić, and M. H. J. Munk, Front. Sys. Neurosci. 8, 108 (2014).

[52] M. Abramowitz and I. A. Stegun, Handbook of Mathematical Functions with Formulas, Graphs, and Mathematical Tables (Dover, New York, 1974).

[53] D. Oakes, J. Appl. Probab. 12, 69 (1975).
[54] E. Bacry, I. Mastromatteo, and J.-F. Muzy, Mark. Microstruct. Liquidity 1, 1550005 (2015).

[55] X. Gao and L. Zhu, Stoch. Process. Appl., DOI: 10.1016/ j.spa.2017.12.001 (2018).

[56] A. Dassios and H. Zhao, Adv. Appl. Probab. 43, 814 (2011).

[57] E. Errais, K. Giesecke, and L. R. Goldberg, SIAM J. Finan. Math. 1, 642 (2010).

[58] A. G. Hawkes and D. Oakes, J. Appl. Probab. 11, 493 (1974).

[59] J. Møller and J. G. Rasmussen, Adv. Appl. Probab. 37, 629 (2005).

[60] P. Dayan and L. Abbott, Theoretical Neuroscience: Computational and Mathematical Modeling of Neural Systems (MIT Press, Cambridge, Massachusetts, 2001).

[61] V. Priesemann, M. H. Munk, and M. Wibral, BMC Neurosci. 10, 40 (2009).

[62] G. Hahn, T. Petermann, M. N. Havenith, S. Yu, W. Singer, D. Plenz, and D. Nikolic, J. Neurophysiol. 104, 3312 (2010).

[63] A. Levina and V. Priesemann, Nat. Commun. 8, 15140 (2017).

[64] A. Clauset, C. R. Shalizi, and M. E. J. Newman, SIAM Rev. 51, 661 (2009).

[65] S. V. Aksenov, M. A. Savageau, U. D. Jentschura, J. Becher, G. Soff, and P. J. Mohr, Comput. Phys. Commun. 150, 1 (2003).

[66] Didier Sornette, J. Phys. I France 4, 209 (1994).

[67] J. A. Bonachela and M. A. Muñoz, J. Stat. Mech. (2009) P09009.

[68] J. A. Bonachela, S. De Franciscis, J. J. Torres, and M. A. Munoz, J. Stat. Mech. (2010) P02015.

[69] W. L. Shew, H. Yang, S. Yu, R. Roy, and D. Plenz, J. Neurosci. 31, 55 (2011).

[70] T. Vogels and L. Abbott, J. Neurosci. 25, 10786 (2005).

[71] A. Kumar, S. Schrader, A. Aertsen, and S. Rotter, Neural Comput. 20, 1 (2008).

[72] A. Levina, U. Ernst, and J. M. Herrmann, Neurocomputing; Variable Star Bulletin 70, 1877 (2007).

[73] F. Droste, A.-L. Do, and T. Gross, J. R. Soc. Interface 10, 20120558 (2013).

[74] T. Wang, M. Bebbington, and D. Harte, Ann. Inst. Stat. Math. 64, 521 (2012).

[75] E. Lewis, G. Mohler, P. J. Brantingham, and A. L. Bertozzi, Secur. J. 25, 244 (2012).

[76] H. Kim, Ph.D. thesis, University of California, Berkeley (2011), https://escholarship.org/uc/item/8nc0r19n.

[77] F. Schoenberg, M. Hoffmann, and R. Harrigan, arXiv: 1703.08202 . 